\title{
Stainless Steel Composite Integrated in Modern Facade
}

\section{Engineering}

\author{
Marc Tulke ${ }^{1}$, Jennifer Watzke ${ }^{1}$, Michael Schomäcker ${ }^{1}$, Alexander Brosius ${ }^{1}$, Janine Bach ${ }^{2}$ and Helmut Hachul ${ }^{2}$ \\ 1. TU Dresden, Institute of Manufacturing Technology, Chair of Forming and Machining Processes, Dresden 01062, Germany \\ 2. University of Applied Science and Arts Dortmund, Architecture and Metal Construction, Dortmund 44227, Germany
}

\begin{abstract}
This paper shows the results of characterising a stainless steel composite material for applications in facade engineering. A number of possible shapes generated with pneumatic stretch forming are presented as a result of simulation studies. In this context a model of a possible facade combined from different shapes is created to show the optical effect of the simulated shapes. Additionally, the manufacturing of a device for pneumatic stretch forming is discussed and first results of transforming the stainless steel composite material with pneumatic stretch forming are shown.
\end{abstract}

Key words: Composite, design, facades, lightweight, pneumatic forming.

\section{Abbreviation}

PE

PMS

polyethylene

pneumatic stretch forming

\section{Introduction}

Due to characteristics like uncoated brightness and a wide variety of surface patterning stainless steel is often used for claddings [1]. Owing to the minimal corrosion of stainless steel, the brightness of the steel boards survives in an urban atmosphere. Other properties like the weight, high material costs and special demands on processing restrict the application of stainless steel in facade engineering [2]. The request for large sized steel boards with several form elements and hidden fasteners in rear ventilated claddings results in certain challenges, e.g., difficulties in forming or high material costs and heavy boards due to increasing material thickness. For optical and ecological reasons stainless steel composites represent an opportunity for application in claddings. The composite consists of two thin and strong surface layers and the thick and light core. The advantages of composite materials include weight reduction

Corresponding author: Marc Tulke, Dipl.-Ing., research field: forming processes. E-mail: marc.tulke@tu-dresden.de. compared with solid material, acoustic and thermal insulation, vibration damping and impact absorption [3].

A composite with aluminium alloy as face material and polyethylene as core is commonly used for claddings. The aluminium composite is resistant to corrosion and can be shaped liked sheet metal. It can be formed by bending and folding with a surface milling process [4]. These techniques enable generating free formed shapes with sharp edges. The stainless steel composite can be machined with same techniques but with the unique design aspects of stainless steel. Currently, free formed surfaces with smooth transitions are favored in facade engineering $[5,6]$. A possible approach for visual upgrading of facades is rear ventilated claddings. The composite will be formed with PMS (pneumatic stretch forming). This process allows the creation of individual surfaces with soft curves. The characterization of the composite material and the realization of the pneumatic stretch forming take place within the project: EVeFA-Stainless-Steel-Composite-Cladding. The executing research centers are the University of Technology Dresden and the University of Applied Science and Arts Dortmund. Aim of the project is the 
application of stainless steel composites in facades. A case study of possible facades is shown in Fig. 1. Based on the light incidence and the different orientations of the form elements a different look arises across the facade.

\section{Characterisation}

The examined stainless steel composite board has a thickness of $4 \mathrm{~mm}$. The face material is $0.4 \mathrm{~mm}$ thick and made of 1.4404. The back side of the composite material has a bright annealed surface (called 2R finish) and the front side has a patterned surface. The fire-retardant core material is a particle reinforced polyethylene with a thickness of $3.2 \mathrm{~mm}$.

To characterise the stainless steel composite material and the three single layers tensile test and bulge test were done. The tensile test, according to DIN EN ISO 6892 [7], is used to determine the characteristic values and the flow curves which is primarily used to describe the material behaviour of the stainless steel composite in simulation model. In tensile test of the composite material first cracks occur in the interlayer at an engineering strain of $10 \%$ that grow during the course of the test while the number of cracks increases. The cracks run from the edge to the middle of the specimen (Fig. 2). Consequently, the face material delaminates the beginning from the edge of the tensile specimen. The properties of the composite material and the single layers are listed in Table 1.

Comparing the properties of the two steel sheets no marked difference is recognized. The stress-strain curves and the characteristic values are similar. The fracture strain of the core is about $5 \%$ and no necking is detected. Therefore, the core material tolerates higher loads in the compound than as single layer.

The bulge test is a preliminary test for stretch forming with a working medium. The testing material is clamped between the blank holder and the die and is loaded with an oil pressure. The inner diameter of the blank holder is $100 \mathrm{~mm}$ and the testing speed is

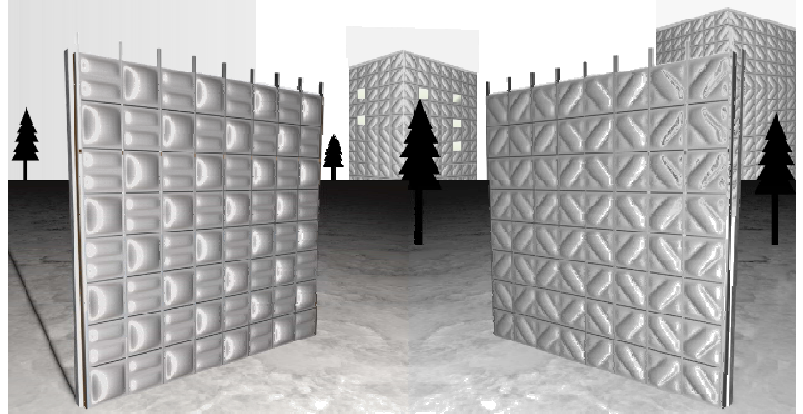

Fig. 1 Case study of facade elements manufactured with PMS.

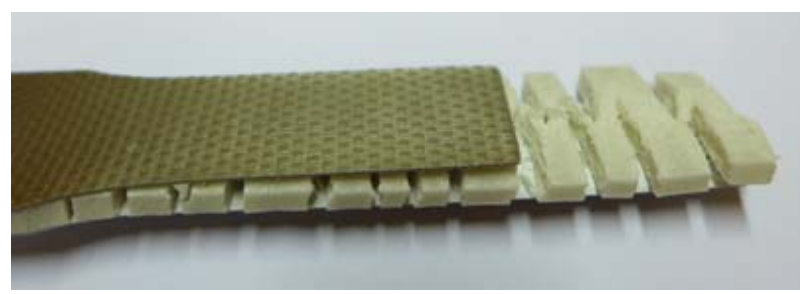

Fig. 2 Tensile specimen of the composite.

Table 1 Properties of face material, core, composite.

\begin{tabular}{lllll}
\hline & 2R & 2R, patterned & PE & Composite \\
\hline $\mathrm{E} / \mathrm{MPa}$ & 173 & 178 & 18 & 39 \\
$\mathrm{R}_{\mathrm{p} 0.05} / \mathrm{MPa}$ & 260 & 249 & 1.4 & 61 \\
$\mathrm{R}_{\mathrm{p} 0.1} / \mathrm{MPa}$ & 273 & 254 & 2,4 & 63 \\
$\mathrm{R}_{\mathrm{p} 0.2} / \mathrm{MPa}$ & 285 & 273 & 4,0 & 65 \\
$\mathrm{R}_{\mathrm{m}} / \mathrm{MPa}$ & 621 & 614 & 13 & 129 \\
$\mathrm{~A}_{\mathrm{g}} / \%$ & 43 & 42.5 & 3.5 & 29.7 \\
$\mathrm{~A} / \%$ & 49.5 & 43.2 & 5.0 & 37.8 \\
r-value & 0.66 & 0.86 & - & 0.36 \\
n-value & 0.22 & 0.22 & - & 0.1 \\
\hline
\end{tabular}

$1.5 \mathrm{~mm} / \mathrm{s}$. At the time of the crack the height of the bulge of the composite material has reached about 38 $\mathrm{mm}$. The bulge of the single sheet is about $33 \mathrm{~mm}$ high. The major and minor strain determined in the bulge tests are presented in Fig. 3. Fig. 4 shows the bulge sample of the composite after failure. The crack occurs centred in the dome and runs in a very straight line. Due to the forming process no wrinkling occurs. The sample of the face material is similar to the composite sample.

The simple geometry of the bulge in Fig. 4 itself shows different reflections of the light in one composite board. Especially, the transitions and the imprint of the bead form expressive visual effects. Based on the bulge test principal aesthetical and 


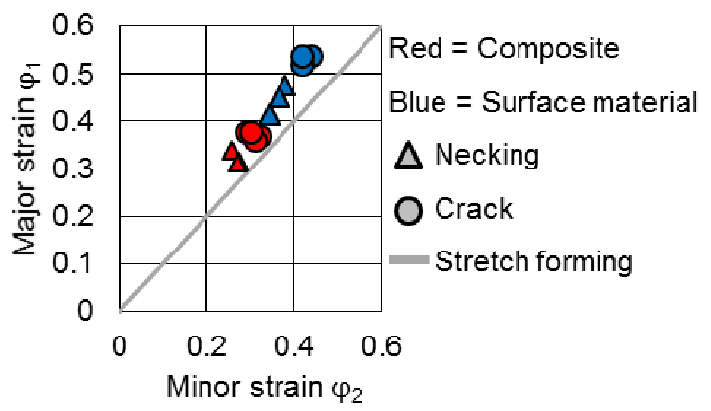

Fig. 3 Bulge test of composite and face material.

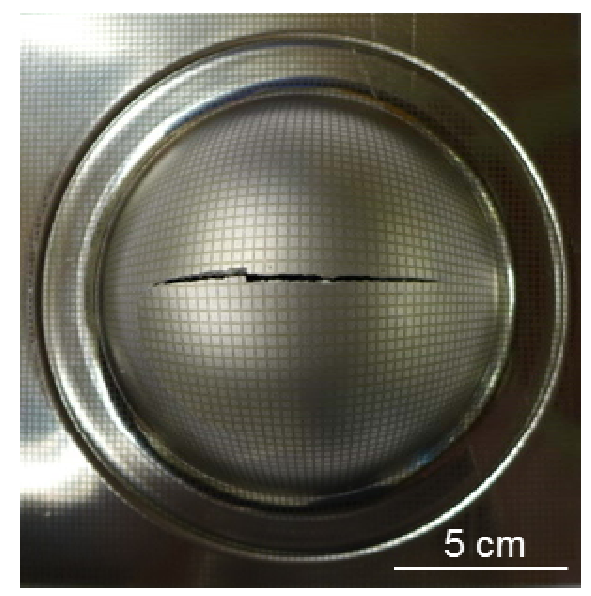

Fig. 4 Bulge test of composite material.

technological demands on the composite can be met. A major condition for using the composite in facade engineering is the firm bond between the face material and the core in the transformed composite board. During all bulge tests no delamination is detected when a crack occurs. The transformed composite boards are cut into segments to identify a possible delamination. In all specimens no disconnection between the core and face materialis detected (Fig. 5). The favoured round transition from the non-transformed composite board to the form element is clearly recognisable in this figure. Another important demand is the flat non transformed part of the composite board that is necessary to fix the transformed boards in the facade. Also this fact is fulfilled as shown in Figs.Fig. 4 and Fig. 5.

To display the strength of the composite material in contrast to the single sheet the tensile force-displacement diagram is presented in Fig. 6. This clearly shows that the tensile force of the

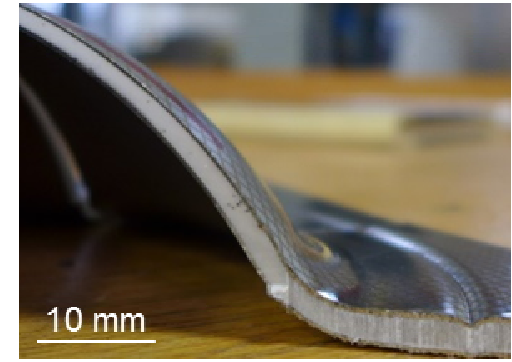

Fig. 5 Test of delamination in composite material.

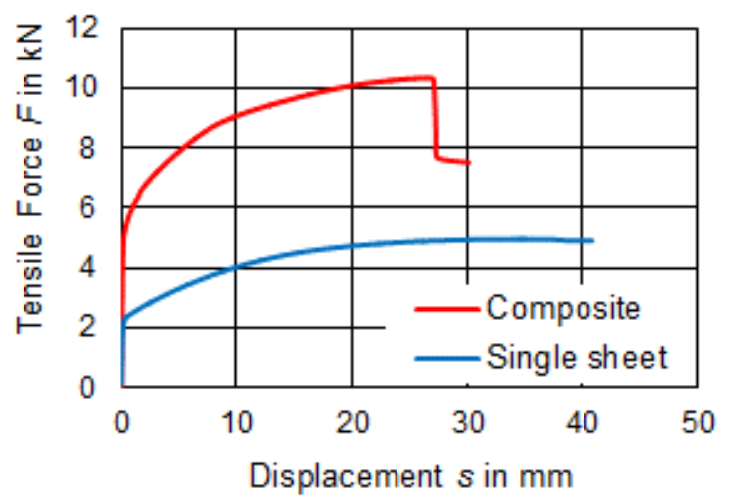

Fig. 6 Force-displacement-diagram.

composite is more than twice as high as the amount of the single sheet. The tensile force of the composite is $10.35 \mathrm{kN}$ and the tensile force of the single sheet is about $4.97 \mathrm{kN}$.

\section{Simulation}

To estimate the formability of the stainless steel composite material in combination with pneumatic stretch forming and to examine potential geometries that can be formed the process is simulated with Simufact. Forming GP 11.0 (regular mesh, implicit code). To evaluate the reliability of the numerical results the bulge test is simulated, too. Comparing the numerical and experimental tests, similar results are attained. For the bulge test the height of the dome is about $38 \mathrm{~mm}$ when crack occurs and in simulation the height is about $33.6 \mathrm{~mm}$ for the same major strain. The difference can be attributed to the material law of the core material because the material law for the composite is tailored towards metal. In this case the non-linear material behavior of the plastic interlayer is neglected. Besides the properties of the composite 
material are determined in tensile test but the material behaviour under biaxial stress condition specifically of the plastic is much different.

In the following figure two possible shapes are shown. The basic form element produced with pneumatic stretch forming is a bulge. The active area of the air pressure in principle is $1 \mathrm{~m}^{2}$ which can be changed with tool inserts and in result different shapes can be created. The air pressure is 6 bar. The necessary die and optional tool insert to generate the presented form elements are shown next to the shapes. The height of the dome is about $120 \mathrm{~mm}$ in case of using no tool insert (Fig. 7a). Fig. 7b shows a more complex shape generated with one tool insert and a maximum dome height of the two symmetric bulges of about $54 \mathrm{~mm}$.

The simulation of pneumatic stretch forming is also used to identify the reaction forces that have to be absorbed by the device. Altogether, the simulation of the pneumatic stretch forming indicates a good formability of the stainless steel composite with this process.

As part of simulation studies a possible advantage with respect to the stiffness of transformed composite boards compared with flat boards was examined. In this connection the displacement as consequence of

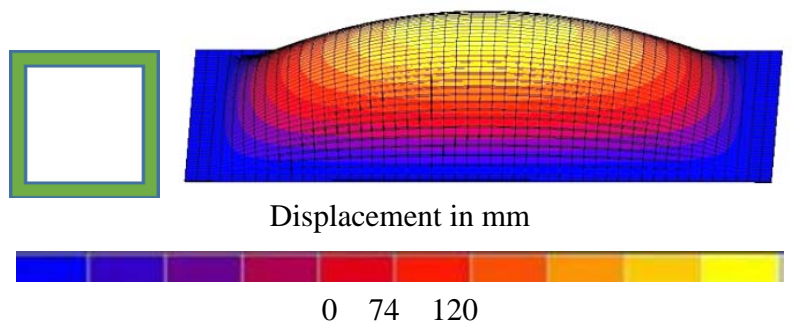

(a) Stretch forming without tool inserts

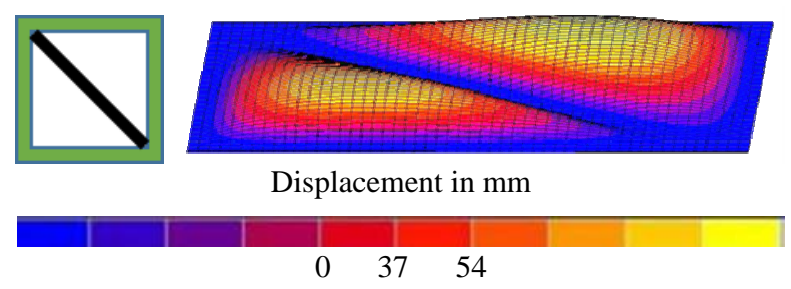

(b) Stretch forming with tool insert

Green $=$ die $\quad$ Black $=$ tool insert

Fig. 7 Composite boards formed with PMS.

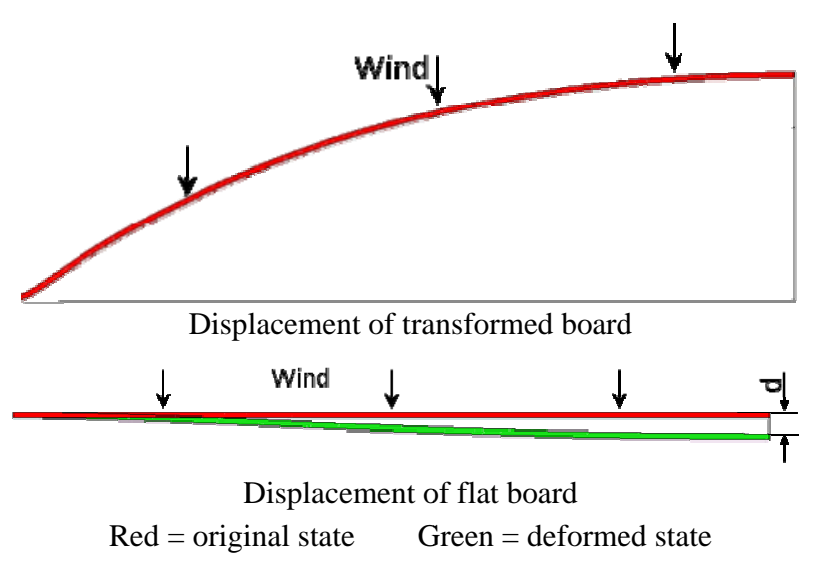

Fig. 8 Displacement due to wind load.

wind equal to blasts near the coast is simulated. The displacement of the transformed composite board due to the wind tends to zero while the flat board has a maximum displacement of $3.6 \mathrm{~mm}$ (Fig. 8). So a higher stiffness is caused by the geometry of the composite board. In both cases the deformation is completely elastic strain, so there is no remaining deformation after the impact of the wind. The simulated boards have an area of $1 \mathrm{~m}^{2}$ and the examined transformed board is shown in Fig. 7a. Because of symmetry only half of the cross section is displayed. For a better recognisability in Fig. 8a scaling factor of 3 is used to show the displacement.

\section{Design Elements in Architecture}

A rear ventilated facade is a self-supporting wall construction system used for chamber forming which is described in DIN 18516 [8]. It consists of different layers of specific materials in order to meet several requirements such as separating inside from outside, stability, natural lighting and ventilation, thermal and sound insulation (Fig. 9) and last but not least it has to give the building a good look. Especially, for constructing economic and sustainable high-end real estates rear ventilated facades are first choice.

Particularly a variety of cladding elements that give the building its unique look are commercially available. Common materials like steel, stainless steel, aluminium, copper and zinc are applied. These range of materials with its characteristics and its own 


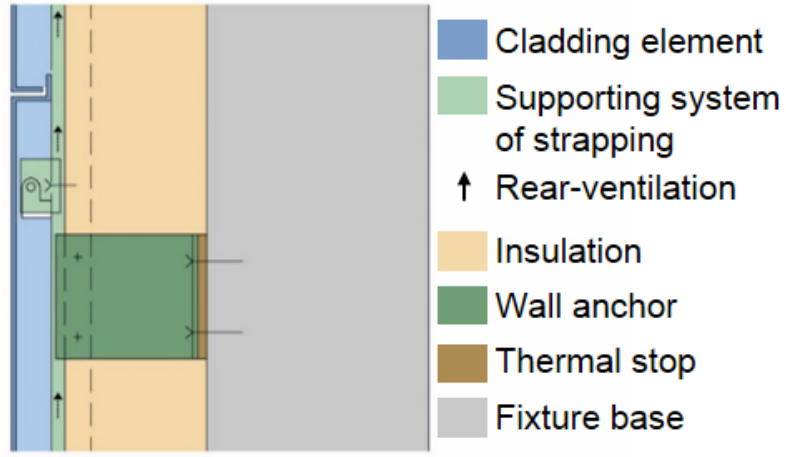

Fig. 9 Concept of rear ventilated cladding.

surfaces are complemented by metal composite materials like the new stainless steel composite material.

The simplest type of cladding elements are tailored pieces of the stainless steel composite which can be fitted to the supporting system by screwing, riveting and bonding. A higher aesthetic value can be reached by folding tailored pieces to tray panels and suspend them into a special substructure.

Additionally to the possibilities described above the composite material provide a big advantage. Easy and complex folded shapes can be constructed by milling the backside of the composite material. This milled line causes a planned weakening of the composite material, so that manufacturers are able to fold them into panels by hand. In this way complex three-dimensional figures can be easily and economically produced (Fig. 10).

In contrast to the folding method which creates sharp edged shapes, it is possible to create slightly and soft curved cladding designs by using pneumatic stretching. The following example shows the visualisation of a possible design which is arranged as a rear ventilated facade. The single composite board of this example is already presented as result of simulation in Fig. 7a.

The element describes a square facade element whose centre bulges out smooth and gentle. Arranged to a facade it could be associated with quilted fabrics. The gleaming surface of the stainless steel composite material emphasizes the variation of light and shade which arises by its three-dimensional shape.

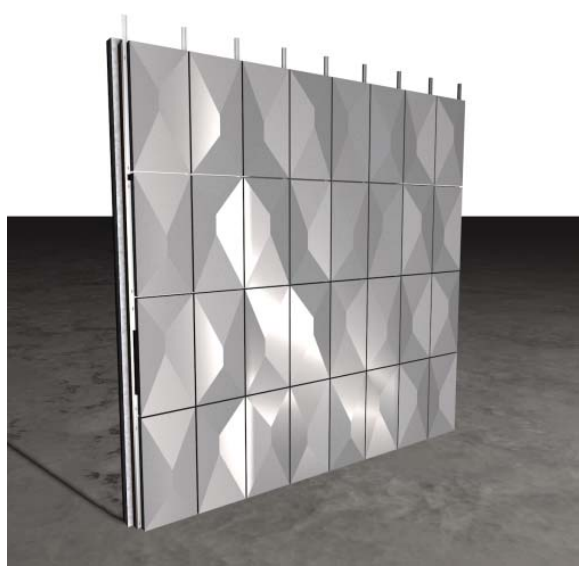

Fig. 10 Facade with folded shapes.

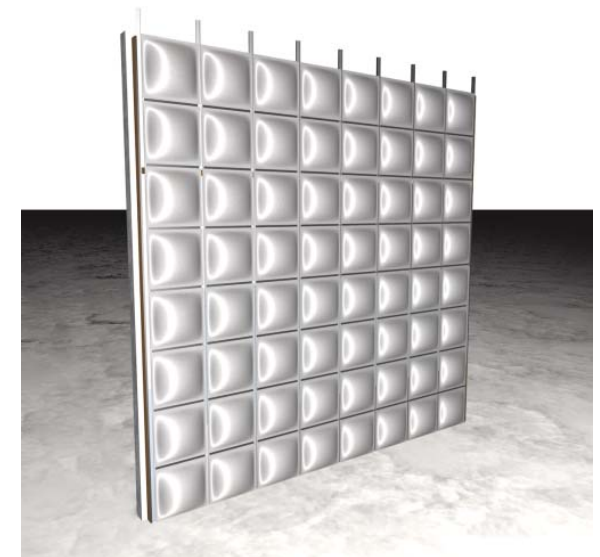

Fig. 11 Example facade 1.

The shown examples of facades demonstrate the potential of the composite with regard to designing facades. Especially, the different reflective properties depend on the form elements in a surface.

\section{Pneumatic Stretch Forming}

Stretch forming describes the forming of metal sheets while the material flow is suppressed due to the clamped blanks [9]. In contrast to the stretch forming with stiff stamps the pneumatic stretch forming uses air pressure to transform the blank. Examinations on sheet metal forming with working media were already done during SPP 1098 with the aim of a homogeneous material allocation for preforming deep drawing shapes [10]. Due to lower elastic properties of the composite material pneumatic stretch forming was chosen to avoid delamination between the two 
material components as a result of material flow. The real flow properties of the combined components have to be determined. The main principle of the pneumatic stretch forming process is shown in Fig. 12.

The demand for new shaping methods for ventilated claddings can be met with the pneumatic stretch forming. This forming technology allows individual round shapes with smooth transitions between the different form elements. With a pressure up to 12 bar large boards of about $1 \mathrm{~m}^{2}$ can be transformed. The form elements can be varied through tool inserts in the bottom part of the tool, shown in Fig. 12. One major advantage of forming with pressurized air is the intact surface quality of the parts, because there is no direct contact with the tool. Therefore, the parts can be assembled without further surface treatment or added process steps. This is limiting waste, because the formed blanks already have the size needed for the facade.

As a result of simulating the pneumatic stretch forming process the forming forces were determined. Based on the simulation results using a blank size of $1.2 \times 1.2 \mathrm{~m}^{2}$ and an active area of the air pressure of about $1 \mathrm{~m}^{2}$ a tool is constructed, as shown in Fig. 13.

The tool consists of an upper and lower tool part. The design is based on the use of a 1,000 kN hydraulic press to provide the clamping forces. The upper part builds a hermetical sealed space to generate

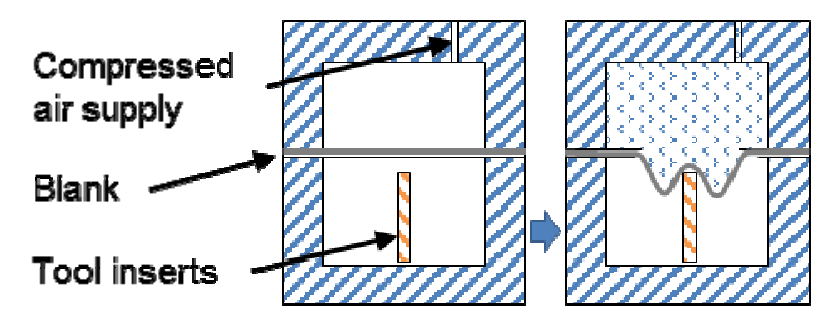

Fig. 12 Main principle of pneumatic stretch forming.

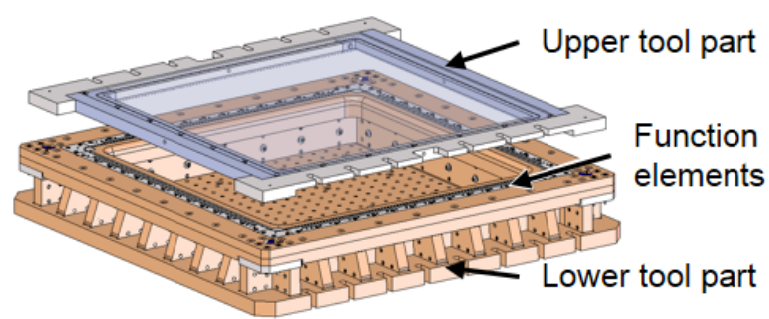

Fig. 13 Construction of pneumatic stretch forming tool. the air pressure for forming the blank clamped between the upper and lower tool part (Fig. 13). The bottom tool part stabilizes the forming and creates a space for the formed composite board. A variation of forming shapes can be created by inserting additional tool inserts into the lower part of the tool. These modules are variable in form and size and can be combined for added variability. In order to maximize formability, the goal is to ensure a homogeneous stretching of the material. In an effort to optimize the stretch behavior different kinds of beads will be examined. For this purpose a modular bead holder is mounted on the clamping surface of the tool. The forces on the bottom part of the tool are very low but due to the size needed for the large blanks it will be very heavy if conventionally made of steel. Modern lightweight design offers the possibility to use a variety of materials in an intelligent construction to reduce weight. In this case, for the lower tool part that is not subjected to heavy loads, a beech tree wood was chosen to substitute steel, thereby reducing its weight by over $50 \%$. Consequently, handling and working with the tool will be much easier in a laboratory environment. Additionally, the tool costs are reduced due to lower material prices and machining costs. The lower part is made of wood whereas only function elements are made of steel.

Due to detailed material studies a complex and variable tooling design could be investigated. With this tool different shapes of facade elements can be produced.

\section{Experimental Procedure}

Based on the numerical and empirical examinations of the new stainless steel composite material and pneumatic stretch forming the tool is manufactured and first tests are done. For the tests the stainless steel composite described in section 0 is used. For the first test the pressure is about 6 bar and the blank holder force is about $500 \mathrm{kN}$. The stainless steel composite board is transformed without the usage of tool inserts. Therefore, the result is a big bulge growing from the 
centre of the composite board. The composite board before and after the pneumatic transformation still in the tool is shown in Fig. 14.

The transformed composite board shows a smooth transition from the non-transformed even part of the blank to the bulge. Also the corners of the big bulge have homogenous radii. The surface of the face material shows no signs of tool contact and has kept a bright metal surface as before the transformation. No scratches or other defects can be detected in the range of the die radius, either. The height of the bulge reaches $113 \mathrm{~mm}$ in good accordance with the results of the numerical tests that predicted a dome height of $120 \mathrm{~mm}$. The resulting difference is only about $6 \%$, but with a small part of material flow that assists the forming of the bulge. Therefore, the dome height will be a little bit less when eliminating the material flow. In Fig. 15, the effects of the different light incidences across the shape are clearly visible and indicate the new design possibilities. The two main prerequisites for the application of the stainless steel composite material in combination with pneumatic stretch forming in facade engineering are the possibility to fasten the boards to facades and prevention of delamination during the forming. For that reason the non-transformed part of the composite board needs to remain flat. In the range of the beads the composite board is flat and no delamination of the face material exists.

When looking at the outer edges of the composite board, however, a material flow from outside of the beads can clearly be recognised. This material flow needs to be suppressed because it can result in wrinkles. Due to high wrinkling, the core material can suffer cracks, because of its lower formability compared to the face material. So the blank holder force has to be increased to suppress the material flow and to avoid wrinkling and realize a stretch forming process.

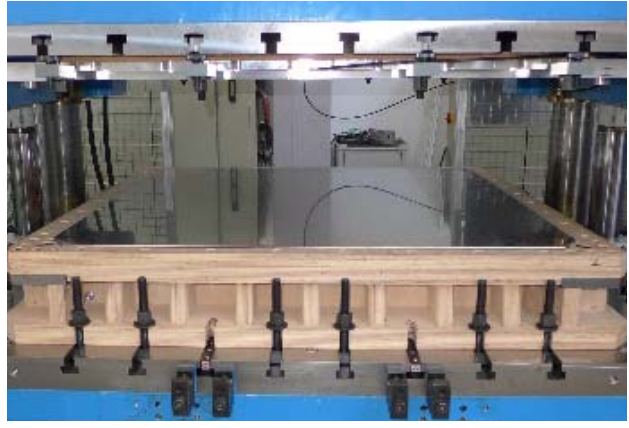

(a) Composite board bevor the forming process

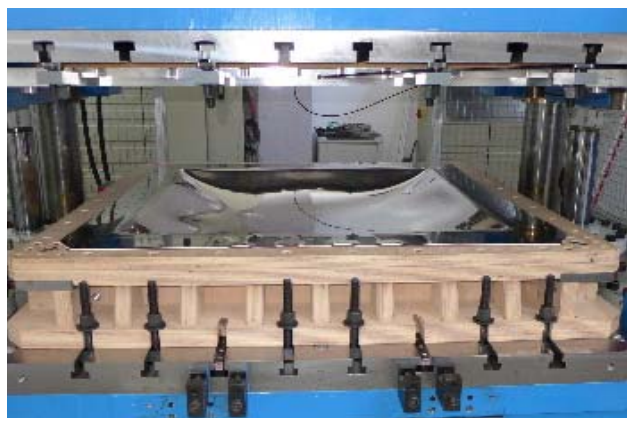

(b) Composite board after the forming process

Fig. 14 Composite board (a) before and (b) after pneumatic stretch forming without tool inserts.

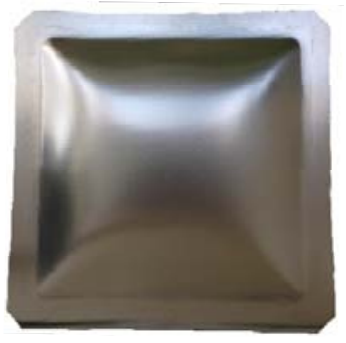

(a) Front side

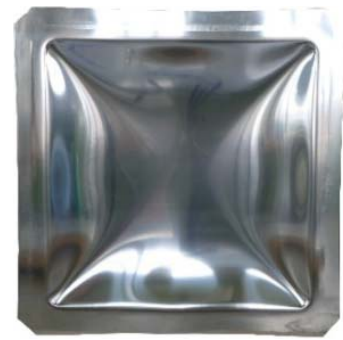

(b) Back side
Fig. 15 Transformed stainless steel composite board.

\section{Conclusions}

The characterisation of the composite material shows a good formability of the stainless steel composite with pneumatic stretch forming. Comparing the tensile test and the bulge test, the formability of the composite depends on the stress condition. The results of the simulation show that complex shapes can be generated. In total the numerical and experimental results indicate that the stainless steel composite combined with the pneumatic stretch forming meet the requirement of modern facade engineering and designing. Shapes with soft curves and different 
reflective properties in one surface can be created. No delamination of the composite material occurs during bulge test of the composite material. In pre-test of pneumatic stretch forming only small areas at the outer edges of the composite board display some delamination effects. The compound of the whole composite board survives even if the specimen fails in bulge test or delaminates in very small parts of a $1 \mathrm{~m}^{2}$ big composite board. This property represents a decisive factor for application in facade engineering.

\section{Outlook}

Additional tests of pneumatic stretch forming will be done mainly with different tool inserts. In this way, different shapes will be created and subsequently investigated regarding their influence on the material flow of the stainless steel composite. It is planned to examine different geometries of beads and to determine their influence on the material flow. Based on these future results a mock-up will be produced for presenting the possibilities of pneumatic stretch-forming. Establishing the theoretical and practical limits of this concept, a design catalogue with different shapes will be compiled.

\section{Acknowledgments}

The presented results are based on the investigations of the project
"EVeFA—Stainless-Steel-Composite-Cladding”, which is kindly supported by the Research Association for Steel Application (FOSTA).

\section{References}

[1] Euro Inox. 2005. Guide to Stainless Steel Finishes, 3rd edition. Building Series, Volume 1.

[2] Hachul, H. 2013. Building Envelopes with Stainless Steel, Stahl und Eisen, VerlagStahleisen GmbH Düsseldorf, fourth ed., pp. 61-7.

[3] Zenkert, D. 1997. An Introduction to Sandwich Construction, EMAS Publ.

[4] 3A Composites GmbH. 2014. ALUCOBOND, Processing and Technical Data, 2nd edition.

[5] Franke, A. 2011. Vortrags-Campus: Neues Denken für Nachhaltiges Bauen, Gebäudehüllen aus Stahl und Glas, Internationale Projekte der Stahlarchitektur, Messe Bau. (In German)

[6] Lother, K. 2013. Integrale Planung im Zusammenspiel von Architekt und Fassadenbauer, DBZ, Fassade.

[7] DIN EN ISO 6892-1. 2009. Metallic Materials-Tensile Testing, Method of Test at Room Temperature.

[8] DIN 18516-1. 2010. Cladding for External Walls_-Part 1: Requirements, Principles of Testing.

[9] DIN 8585-2. 2003. Manufacturing Processes under Tensile Conditions_-Part 2: Stretch Reducing; Classification, Subdivision, Terms and Definitions.

[10] Kleiner, M., Homberg, W., and Trompeter, M. 2006. Hochdruckumformung einzelner Bleche, Abschlussbericht zum DFG-Schwerpunktprogramm SPP1098. (In German) 